\title{
Cross-Tree Adjustment for Spatialized Audio Streaming over Networked Virtual Environments
}

\author{
Ke Liang \\ School of Computing \\ National University of Singapore \\ Singapore 117417 \\ liangke@comp.nus.edu.sg
}

\author{
Roger Zimmermann \\ School of Computing \\ National University of Singapore \\ Singapore 117417 \\ rogerz@comp.nus.edu.sg
}

\begin{abstract}
In recent years, integrated spatialized voice services have become an appealing application for networked virtual environments (NVE), e.g., Second Life. With a spatialized voice service, people can identify who is talking if there are several participants in the vicinity. The key challenge in a spatialized audio streaming application is how to disseminate audio streams while observing bandwidth limits of end-user computers and tight latency constraints, and it can be modeled as NP-complete problem. In this paper, we propose a heuristic algorithm called CTA for spatialized audio streaming over NVEs in a peer-to-peer manner. The proposed algorithm was applied to real avatar mobility traces collected from Second Life, and the simulation results demonstrate that (a) CTA can achieve a high ratio of successful to candidate receivers and (b) CTA enables most of the successful receivers to enjoy minimum latency.
\end{abstract}

\section{Categories and Subject Descriptors}

H.4.3 [Information Systems Applications]: Communications Applications - Computer conferencing, teleconferencing, and videoconferencing

\section{General Terms}

Algorithms, Measurement, Performance

\section{Keywords}

Proximity audio, spatial audio, peer-to-peer streaming, application level multicast

\section{INTRODUCTION}

Networked virtual environments (NVE) are large-scale interactive applications in which users can move their avatars in a shared virtual world while interacting with the environment and other online users. One of the most well-known and successful NVE's is Second Life, which is an online 3D virtual world built by its residents.

Permission to make digital or hard copies of all or part of this work for personal or classroom use is granted without fee provided that copies are not made or distributed for profit or commercial advantage and that copies bear this notice and the full citation on the first page. To copy otherwise, to republish, to post on servers or to redistribute to lists, requires prior specific permission and/or a fee.

NOSSDAV'09, June 3-5, 2009, Williamsburg, Virginia, USA.

Copyright 2009 ACM 978-1-60558-433-1/09/06 ...\$5.00.
The goal of voice services provided in a NVE is to enable users to speak to each other naturally and seamlessly. Some existing systems (e.g., Skype and AudioPeer [9]) provide monophonic voice services that are separate from any NVE, while others aim to provide a more integrated approach. The most important disadvantage of existing monophonic audio systems is that they are not designed to utilize the spatial information (e.g., location, distance and directionality) between avatars. However, with a spatialized voice service, users can more easily identify who is speaking if several people surround them. By matching users' aural environment to their visual perception more immediacy and dynamism is lent to their interaction with others, providing an overall more immersive experience.

Spatialized 3D audio rendering is now available on PCs with the help of software libraries (e.g., OpenAL) that can take advantage of different hardware configurations. 3D audio can be rendered via two or more loudspeakers, or via stereo headphones. Although there are some algorithms that regenerate $3 \mathrm{D}$ audio from only one or two audio streams, most 3D audio processing modules require access to each spatially positioned source and hence multiple audio streams should be kept separate until rendered.

Boustead and Safaei [1] have examined different delivery architectures for audio transmission in large-scale virtual environments, including client-server and peer-to-peer infrastructures. In a client-server topology for $3 \mathrm{D}$ audio transmissions, the central server is responsible for receiving all audio streams directly from all sources (speakers) and forwarding pre-rendered 3D streams to corresponding receivers. 3D audio rendering for all audio streams is accomplished by the central server based on the avatars' spatial information and then encapsulated into stereo streams that are sent back to each receiver. In this approach, the central server requires significant computational resources and a large amount of bandwidth to process and disseminate audio streams. To remove the bottleneck at the central server, some proxy-based solutions [7] have been proposed to balance the bandwidth and processing load [3]. The 3D voice provider of Second Life, Vivox, employs a client-server infrastructure.

By utilizing a peer-to-peer based approach - which can alleviate the scalability problems caused by the client-server design - we disseminate audio streams using applicationlevel multicast trees built on overlay networks that take into consideration heterogeneous bandwidth limits of users and tight latency constraints. Because the upload and down- 
load bandwidths ${ }^{1}$ of each node in a peer-to-peer network are limited and heterogeneous, the task to effectively utilize the network resources when disseminating several separate audio streams while keeping the overlay latency bounded becomes very challenging. Some receivers may not be able to receive the audio streams that they are supposed to (we call those users unsuccessful receivers), and some successful receivers may have a higher latency due to a non-optimal dissemination path of the audio stream. Hence, our research aims to (1) maximize the number of successful receivers of corresponding audio streams and (2) minimize the latency for those successful receivers. The task can be modeled as a well known $\mathcal{N} \mathcal{P}$-complete problem [2]. Recent work exists on heuristic algorithms to construct a degree-bounded minimum average-latency spanning tree for one node $[1,5]$ or all nodes [4].

Previously we have proposed a local tree adjusting (LTA) algorithm [8] designed to maximize the number of successful receivers while keeping the latency for those receivers low. LTA can fully utilize the available network resources for each speaker to construct a multicast tree. However, it lacks fairness to new audio sources since existing ones may have already exhausted network resources. In this paper, we propose a heuristic cross-tree adjustment (CTA) algorithm to solve this fairness issue by constructing and adjusting multiple multicast trees using a greedy approach. The proposed algorithm differs from previous work in that (1) we only construct a multicast tree for a node when it starts speaking and (2) we adjust the new multicast tree with respect to other existing multicast trees who have common nodes with the new multicast tree. The simulation results demonstrate that CTA can outperform LTA both in terms of the number of successful receivers and the ratio of successful receivers that experience minimal latency.

The rest of this paper is organized as follows. Section 2 describes the system model for spatialized audio streaming over NVEs. The problem we are aiming to solve is formulated in Section 3. The proposed algorithm, CTA, is described in detail in Section 4. Section 5 describes the evaluation of our proposed algorithm. Finally, Section 6 draws conclusions of the whole paper.

\section{SYSTEM MODEL}

The users (avatars) in an NVE can move in the system at will and any two users can communicate with each other when their distance is less than $r$, the radius of a node's area of interest (AoI). The AoI is defined as the space around the user where interaction (e.g., voice communication) is likely to happen and it corresponds to the hearing range of each user in our audio streaming scenario. We assume that location and AoI information of each node are maintained at the game server. Existing algorithms and methods exist for this purpose and we do not provide any details here. Every user can speak anytime and at any location. Each user $j$ has a limited upload bandwidth $u_{j}^{\max }$. The upload capacity is measured in units, where 1 unit is defined as the required bandwidth for a single audio stream and measured in bits/s. We will use the terms user, node and avatar interchangeably throughout this paper.

We model the system as a digraph $G=(V, E) . V$ is the set of nodes representing users in the system. $E$ is the set

\footnotetext{
${ }^{1}$ We only consider upload bandwidth limits in this paper.
}

of edges. An edge $e(i, j)$ indicates that node $n_{i}$ is willing to send (if $n_{i}$ is a speaker) or forward an audio stream to $n_{j}$. The nodes within the AoI of node $n_{i}$ are called neighbor nodes of $n_{i}$. Every user periodically exchanges information (i.e., bandwidth usage and end-to-end latency) with its neighbor nodes. When a node starts speaking, all its neighbor nodes are candidate receivers for the audio stream sent from the speaker. Due to the bandwidth limits of the neighbor nodes and the speaker, only a fraction of the candidate receivers, called successful receivers, may actually be able to receive the stream.

Transmission of any audio stream is restricted within the AoI of the speaker who sent the stream. That is, all nodes outside of the hearing range of the speaker will not receive or forward the audio stream. When a node $n_{i}$ starts to speak, it will construct a temporal multicast tree $T_{0}^{i}$ rooted at $n_{i}$. All candidate receivers are members of $T_{0}^{i}$, that is, $T_{0}^{i}$ is initially constructed without considering the bandwidth limits of nodes. $T_{0}^{i}$ is a shortest path tree (SPT) which is a combination of shortest paths from the speaker to all nodes within its AoI.

If some nodes in $T_{t m p}^{i}$ have conflicts ${ }^{2}$ (since one node may be an intermediate node in other existing multicast trees), $T_{0}^{i}$ should be tailored to $T_{s p t}^{i}$ by cutting corresponding edges incident with those conflict nodes. This operation will result in some orphan nodes which are candidate receivers but cannot obtain the audio stream from the speaker. The orphan nodes will be attached to nodes in $T_{s p t}^{i}$ that still have upload bandwidth available, and then the final multicast tree is formed as $M^{i}$. The reason we chose SPT as the basis of the multicast tree is two-folded. First, members of an SPT will benefit from its built-in characteristics, i.e., the latencies from the source to members of SPT are minimal. Second and more importantly, compared to other spanning tree techniques, e.g., a minimum spanning tree or Hamiltonian path, an SPT has less depth and a larger fan-out. Therefore, edge cutting operations will result in less orphan nodes.

Our goal is (in the presence of bandwidth limits of nodes) to maximize the total number of successful receivers while at the same time minimizing the average latency from speakers to their successful receivers. The most critical challenge is that constructing an optimal multicast trees is highly computationally complex as the task is known to be $\mathcal{N} \mathcal{P}$ complete. Next we will formally state the problem and then study its solutions with heuristics.

\section{PROBLEM FORMULATION}

Our objective is to enable many-to-many audio streaming, which requires the construction of a forest that consists of multicast trees rooted at all the speakers in the presence of bandwidth limits of nodes and latency constraints. We define the problem as constructing a forest of degree-bounded minimum latency trees:

$$
\begin{aligned}
\text { Maximize } & :\left|R^{S}\right|=\sum_{i \in S}\left|R_{i}\right| \\
\text { Minimize } & : \frac{1}{\left|R^{S}\right|} \sum_{j \in S} \sum_{k \in R_{j}} L(j, k) \\
\text { S.t } & : \quad u_{x} \leq u_{x}^{m}, m \in V
\end{aligned}
$$

\footnotetext{
${ }^{2}$ In case of the upload bandwidth required for the node exceeds its maximum capacity.
} 
where $R_{i}$ is defined as the set of successful receivers of speaker $n_{i}$, and $S$ refers to the set of speakers. $L(j, k)$ denotes the latency of the path from node $n_{j}$ to node $n_{k}$. The first optimization objective is to maximize the number of successful receivers, subject to nodes' bandwidth limits. Once the first goal is satisfied, the average latency of those successful receivers achieved by Eqn. 1 is to be minimized. Due to node dynamics, system churn and avatars' free will to speak, it is very challenging to find an optimal solution to the multiobjective problem stated above.

\begin{tabular}{|l|l|}
\hline Term & Definition \\
\hline \hline$M^{i}$ & Multicast tree rooted at speaker $i$ \\
$T_{0}^{i}$ & Temporal multicast tree root at speaker $i$ \\
$T_{x}^{i}$ & SPT deduced by removing leaf nodes from $T_{x-1}^{i}$ \\
$T_{s p t}^{i}$ & SPT root at speaker $i, T_{s p t}^{i}=M^{i} \cap T_{0}^{i}$ \\
$V(T)$ & Set of nodes in the tree $T$ \\
$V_{c}^{i}$ & Set of all nodes in $T^{i}$ that have conflicts \\
$V_{x}^{i}$ & Set of leaf nodes of $T_{x}^{i}$ \\
$D_{j}^{i}$ & Set of descendants of node $j$ in $T^{i}$ \\
$W_{j}^{l}$ & Local weight set of node $j$ in tree $T^{i}$ \\
$W_{j}^{g}$ & Global weight set of node $j$ \\
$f(j)$ & Forward node for node $j$ \\
$u_{j}^{m}$ & Maximum upload units of node $j$ \\
$u_{j}^{s}$ & Used upload units for nodes in SPTs at node $j$ \\
$c_{j}^{i}$ & Number of children of node $j$ in $T^{i}$ \\
\hline
\end{tabular}

Table 1: List of terms used.

Instead of achieving the two objectives sequentially, we try to achieve a compromise between the two concurrently. In our approach, the multicast tree $M^{i}$ for each speaker $n_{i}$ consists of a SPT $\left(T_{s p t}^{i}\right)$ and further orphan nodes attached to it. That is, $T_{s p t}^{i}$ is a subgraph of $M^{i}\left(T_{s p t}^{i} \subseteq M^{i}\right)$. The temporal multicast tree for speaker $n_{i}$ is $T_{0}^{i}$, which is also a SPT. The heuristic is that the larger $\left|M^{i} \cap T_{0}^{i}\right|$ is, the more likely the multicast tree will have both, a high successful receiving ratio and low average latency. Therefore, our proposed heuristic algorithm is to maximize $\left|M^{i} \cap T_{0}^{i}\right|$ for all multicast trees in the system:

$$
\begin{aligned}
\text { Maximize } & : \quad\left|T_{s p t}^{S}\right|=\sum_{i \in S}\left|T_{s p t}^{i}\right| \\
\text { S.t } & : \quad u_{x} \leq u_{x}^{m}, m \in V\left(T_{s p t}^{S}\right)
\end{aligned}
$$

The key challenge of our approach is how to allocate the upload bandwidth of bandwidth-deficient nodes for the multiple SPTs that they are associated with.

\section{APPROACH}

Douceur [4] proposed a approximation algorithm to maximize the total upload capacity by constructing a depth- 2 multicast tree for each node in the system. However, in voice communication applications it is a waste of network resources to maintain the multicast trees that are not in use. Therefore, distribution trees for voice dissemination should only be constructed once speakers start to talk, and be destroyed after the conversation ends.

In our previous approach [8], a multicast tree was constructed independently for each speaker, and a local tree adjusting algorithm (LTA) was applied to modify the multicast tree with respect to the remaining available bandwidth of neighboring nodes. This straightforward approach can be quickly employed at each speaker, but it is unfair to new speakers as the network resources may have been exhausted by existing speakers.

Here we introduce a heuristic method to allocate network resources for all speakers, termed cross-tree adjusting (CTA), which outperforms LTA both in terms of fairness and the total number of successful receivers. In addition, the proposed algorithm can increase the ratio of successful receivers with minimum latency. CTA constructs the multicast trees incrementally using a greedy approach. If a new multicast tree and other existing multicast trees have overlapping nodes then all involved trees will be adjusted to observe the bandwidth limits of all nodes. Furthermore, any multicast tree will be destroyed if the corresponding speaker has kept silent for $t_{m}$ seconds.

A new multicast tree is constructed in two phases. The first step, which is similar to our earlier work [8], involves the constructing of a shortest paths tree (SPT) rooted at the speaker without considering the nodes' upload bandwidth limits. The second step is to re-allocate the upload bandwidth for nodes that have bandwidth conflicts. The proposed algorithm is applied in the second step.

\subsection{Temporal Multicast Tree Construction}

Every node in an AoI periodically exchanges information with its neighbor nodes. Therefore, every node is aware of bandwidth limits/usage of every neighbor node and the endto-end latency between any two neighbor nodes. Once node $n_{i}$ starts speaking, it can quickly construct a temporal SPT $\left(T_{0}^{i}\right)$ for the speaker without considering upload bandwidth limits of its neighbor nodes. The complexity to construct a SPT is $O\left(\left|V\left(T_{0}^{i}\right)\right| \log \left|V\left(T_{0}^{i}\right)\right|\right)$ when a Fibonacci heap is utilized.

After the temporal SPT is constructed, a post-order tree traversal with time complexity $O\left(\left|V\left(T_{0}^{i}\right)\right|\right)$ is applied to $T_{0}^{i}$, marking conflicting nodes and partitioning all nodes into different node sets $\left(V_{j}^{i}, 0 \leq j \leq m\right.$, where $m$ is the length of the longest path in $T_{0}^{i}$ ). Let $T_{1}^{i}$ denote a SPT which is derived by removing all leaf nodes from $T_{0}^{i}$. Through the same reasoning, $T_{x+1}^{i}$ is obtained by removing all leaf nodes from $T_{x}^{i}$, while at the same time those leaf nodes are grouped into $V_{x}^{i}, V_{x}^{i}=V\left(T_{x}^{i}\right) \backslash V\left(T_{x+1}^{i}\right)$. After the postorder tree traversal on $T_{0}^{i}$, all nodes in the AoI of speaker $i$ will be partitioned into node sets $V_{0}^{i}, V_{1}^{i}, \ldots, V_{m}^{i}$, as shown in Figure 1.

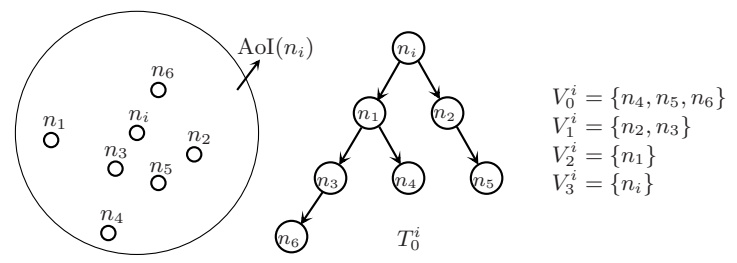

Figure 1: Temporal multicast tree construction and node partitions.

\subsection{Cross-Tree Adjusting (CTA)}

The second step involves the handling of conflicts caused by $T_{0}^{i}$. Some edges in the new SPT $T_{0}^{i}-$ or other existing multicast trees that have overlapping intermediate nodes with $T_{0}^{i}-$ will be removed in this step. The idea is to al- 
locate the upload bandwidth of conflict nodes to maximize $\left|T_{s p t}^{i}\right|$. Differing from the previous algorithm LTA, which allocates upload bandwidth of nodes in the local multicast tree, the proposed algorithm CTA involves all multicast trees that have overlapping conflict nodes with the local multicast tree.

There are two processes for CTA: (1) a voting process, that decides which potential edges need to be removed in the local and other existing multicast trees, and (2) an allocation process, which allocates upload bandwidth for all nodes and modifies the topologies of multicast trees according to the voting results from the first process. We will describe the two processes in detail.

\subsubsection{Voting Process}

Each node in the multicast tree only has one parent node which is responsible for forwarding the audio stream to the node. Once the link between the node and its parent is removed, the node and all its descendant nodes will no longer receive the stream. We define the weight of an edge, $w(e)$, as the maximum number of downstream nodes (descendants) who incur the minimum latency. For example, as shown in Figure 1, $w\left(e\left(n_{3}, n_{6}\right)\right)=1$ and $w\left(e\left(n_{i}, n_{1}\right)\right)=4$ since there is only one downstream node which edge $e\left(n_{3}, n_{6}\right)$ can support while $e\left(n_{i}, n_{1}\right)$ can support four. It should be noted that $w\left(e\left(n_{i}, n_{j}\right)\right)$ depends on the weight of the outgoing edges and the upload bandwidth limits of $n_{j}$. For instance, if $u_{j}^{m}=1$, then $w\left(e\left(n_{i}, n_{1}\right)\right)=3$, hence $e\left(n_{1}, n_{4}\right)$ should be removed from the multicast tree.

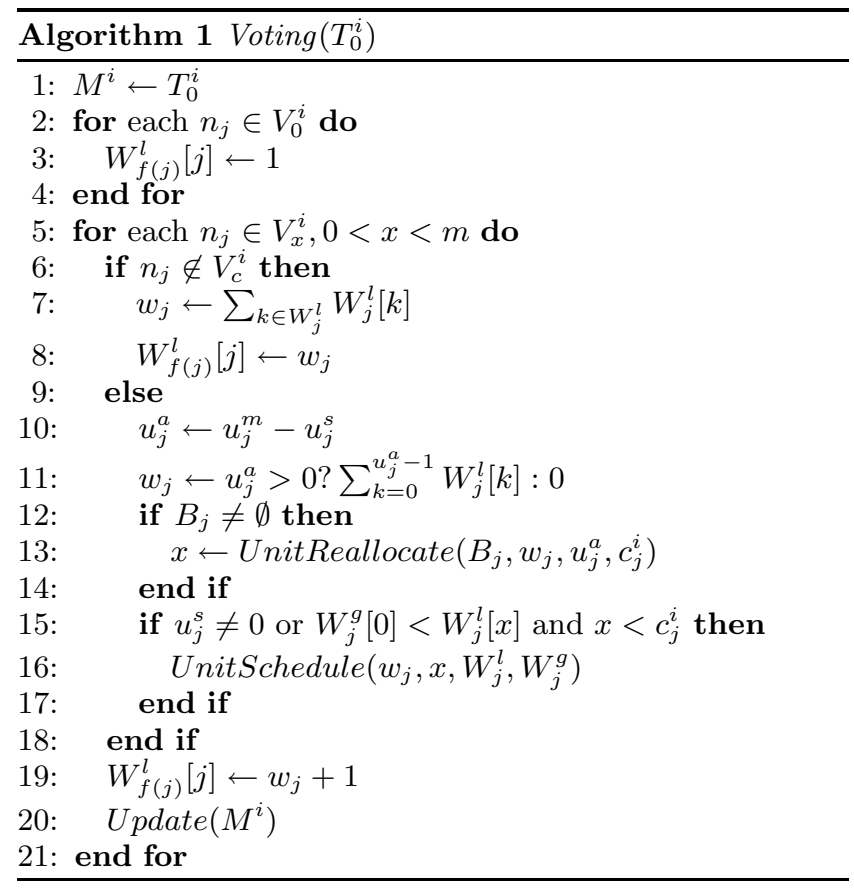

The weight of every edge in $T_{0}^{i}$ is computed at its end vertex, since every node has only one parent in the tree structure. Furthermore, weights of all edges are computed in a bottom-up manner, starting from nodes in $V_{0}^{i}$ to $V_{m-1}^{i}$. Every node $n_{j}$ in the system will have a global weight set (denoted by $W_{j}^{g}$ ) which stores the outgoing edges and their weights in existing SPTs. The size of $W_{j}^{g}$ is determined by its outgoing bandwidth limits. Elements in $W_{j}^{g}$ are sorted from left to right in non-increasing order. Every node $n_{j}$ in new SPT $\left(T_{0}^{i}\right)$ will have a temporal local weight set (denoted by $W_{j}^{l}$ ) which stores the same type of information as $W_{j}^{g}$ but is merged into $W_{j}^{g}$ in the second process. $W_{j}^{g}$ is initialized when the node joins the system and $W_{j}^{l}$ is initialized by the post-order tree traversal in the temporal SPT tree construction step.

The voting process starts from the leaf node set $V_{0}^{i}$, as shown in Algorithm 1 (lines 2-4). In the non-leaf node sets, nodes without conflicts will be processed first. If a nonleaf node $n_{j}$ has no conflicts (Algorithm 1, lines 6-9), it will calculate $w_{j}\left(w_{j}=w\left(e\left(f(j), n_{j}\right)\right)\right)$ and propagate this weight to its parent's local weight set, $W_{f(i)}^{l}$. For a nonleaf conflict node $n_{j}$, all the remaining upload units of $n_{j}$ will be allocated to edges in $T_{0}^{i}$, as shown in lines 10-11 of Algorithm 1. Furthermore, UnitReallocate will re-allocate some units indicated in $B_{j}$ and occupied by other SPTs to $T_{0}^{i} . B_{j}$, which is defined as the backup unit set of $n_{j}$ will be explained later. If all upload units of the conflict node $n_{j}$ are allocated to nodes in SPTs, $w_{j}$ is calculated and propagated to $f(j)$. In the meantime, $M^{i}$ is updated (Algorithm 1, line 19) by removing the remaining edges in $W_{j}^{l}$ and their downstream edges and nodes.

When some remaining local edges have larger weights than edges in $W_{j}^{g}$, the upload unit scheduling algorithm, as shown in Algorithm 2, will be applied to calculate $w_{j}$ and propagate it to the parent's local weight set, $W_{f(j)}^{l}$.

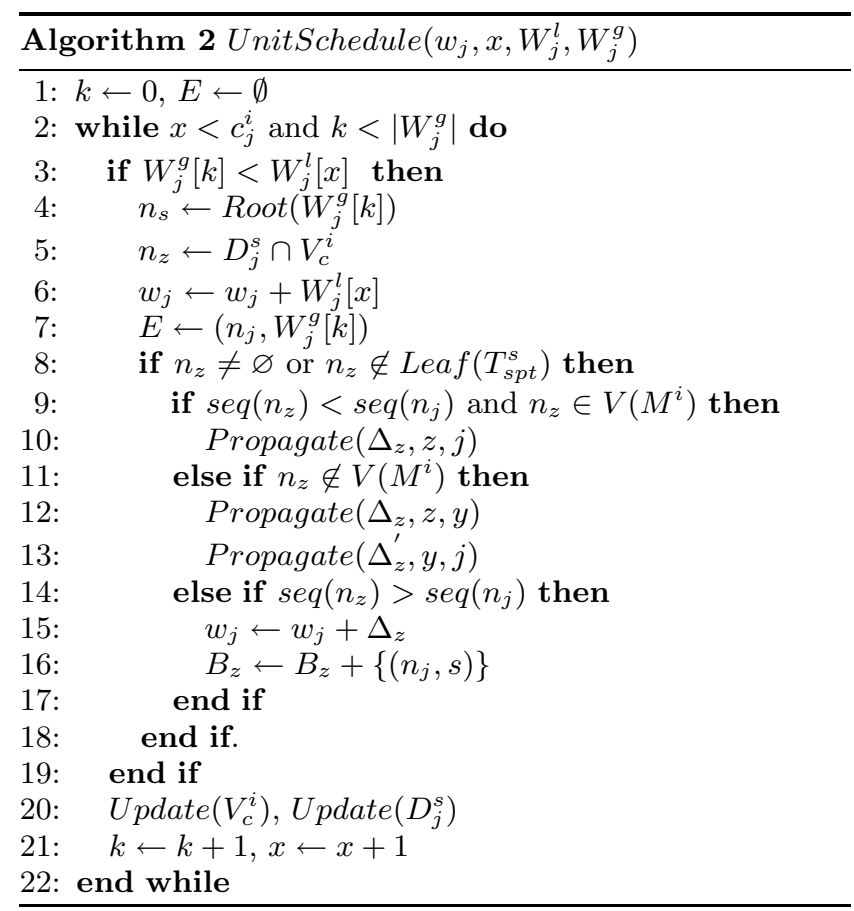

Let $n_{s}$ denote the speaker that has an overlapped conflict node $n_{j}$ with $T^{i}$. Let $n_{z}$ refer to a descendant node of $n_{j}$, both in $T_{0}^{i}$ and $T_{s p t}^{s}$, and $n_{z}$ be a conflict node in $T_{0}^{i}$. If $n_{z}$ does not exist, or $n_{z}$ is a leaf node in $T_{s p t}^{s}$, the upload unit allocated for the edge $e\left(W_{j}^{g}[k]\right)$ will be re-allocated to the edge $e\left(W_{j}^{l}[x]\right.$ ) (Algorithm 2, line 6) since the re-allocation may bring in more receivers that have minimum latency. As a result, a tuple $\left(n_{j}, e\left(W_{j}^{g}[k]\right)\right)$ will be inserted into $E$, the 
set of to-be-removed global edges. $E$ will be used to adjust other multicast trees in the allocation process.

If $n_{z}$ is a forwarder in $T_{s p t}^{s}$, the occupied upload units at $n_{z}$ for $T_{s p t}^{s}$ may be re-allocated for $T_{0}^{i}$. There are three possible cases, as shown in Figure 2, that need to be handled: Case $1, n_{z}$ has been processed in the voting process and it has not been removed from $M^{i}$, case $2, n_{z}$ has been processed in the voting process but it has been removed, and case 3, $n_{z}$ has not been processed in the voting process yet.

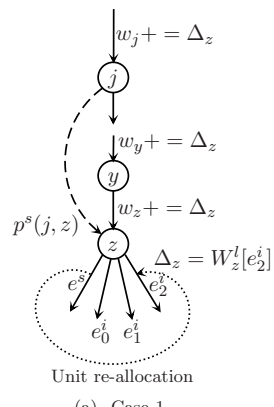

(a). Case 1

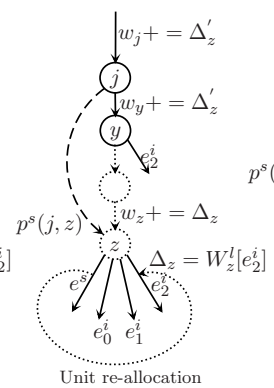

(b). Case 2

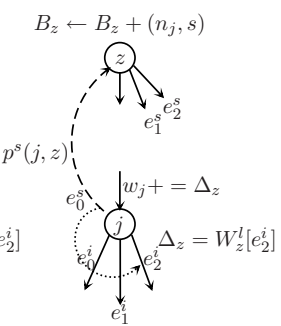

(c). Case 3
Figure 2: Three cases for units scheduling at two conflict nodes, $n_{j}$ and $n_{z}$.

As illustrated in Figure 2, two conflict nodes $n_{j}$ and $n_{z}$ belong to both $T_{0}^{i}$ and $T_{s p t}^{s}$. The node we are processing is $n_{j}$ and $n_{z}$ is a descendant of $n_{j}$ in both SPTs. Let $p^{s}(j, z)$ denote the path from $n_{j}$ to $n_{z}$ in $T_{s p t}^{s}$, and $p^{i}(j, z)$ denote the path from $n_{j}$ to $n_{z}$ in $T_{0}^{i}$. For case 1 (Algorithm 2, lines $9-10)$, the weight of edges in the path $p^{i}(j, z)$ will be updated as follows. First, the units occupied by edges in $T_{s p t}^{s}$ starting from $n_{z}\left(e^{s}\right)$ will be re-allocated to edges in $T_{0}^{i}$ starting from $n_{z}\left(e_{2}^{i}\right)$. Then, we propagate (Algorithm 2, line 11) the increased weight, $\Delta_{z}\left(\Delta_{z}=W_{z}^{l}\left[e_{2}^{i}\right]\right.$ in the example $)$, from $n_{z}$ to $n_{j}$ along the path $p^{i}(j, z)$. The example only shows the case when there is one unit to be re-allocated. A multiple units re-allocation works analogously.

For case 2 (Algorithm 2, lines 11-13), the increased weight $\Delta_{z}\left(\Delta_{z}=W_{z}^{l}\left[e_{2}^{i}\right]\right.$ in the example $)$ will be propagated from $n_{z}$ along the path $p^{i}(z, j)$ until $n_{y}$, which is in $V\left(M^{i}\right)$, is reached. The weight of edges between $n_{z}$ and $n_{y}$ will be increased by $\Delta_{z}$ (Algorithm 2, line 12), and the weight of edges between $n_{y}$ and $f(j)$ will be increased by $\Delta_{z}^{\prime}$ (Algorithm 2 , line 13). As shown in Figure 2(b), $\Delta_{z}^{\prime}$ in this example is computed as follows:

$$
\Delta_{z}^{\prime}= \begin{cases}W_{z}^{l}\left[e_{2}^{i}\right]-W_{y}^{l}\left[e_{3}^{i}\right] & \text { if } W_{z}^{i}\left[e_{2}^{i}\right]>W_{y}^{i}\left[e_{3}^{i}\right] \\ 0 & \text { if otherwise }\end{cases}
$$

For case 3 , where $n_{z}$ has not been processed yet, $w_{j}$ is increased by $\Delta_{z}, \Delta_{z}=W_{z}^{l}\left[e_{2}^{i}\right]$. A tuple $\left(n_{j}, s\right)$ will be inserted into the backup unit set of $n_{z}$, denoted as $B_{z} \cdot\left(n_{j}, s\right)$ in $B_{z}$ means that once $n_{z}$ is processed, it can be used to re-allocate units at $n_{z}$ occupied by $T_{s p t}^{s}$ if $n_{j}$ is not removed.

The complexity of unit re-allocation for cases 1, 2 and 3 is $O\left(\left|V\left(T_{0}^{i}\right)\right|\right), O\left(\left|V\left(T_{0}^{i}\right)\right|\right)$ and $O(1)$ respectively. Therefore the voting process is a polynomial algorithm with complexity $O\left(\left|V\left(T_{0}^{i}\right)\right|^{2}\right)$.

\subsubsection{Allocation Process}

After the voting process is completed, a top-down process is applied to allocate units at all nodes in $M^{i}$ and adjust other SPTs according to $E$ generated from the voting process. For every node, $n_{j}\left(n_{j} \in V\left(M^{i}\right)\right)$, the global weight set, $W_{j}^{g}$ will be updated: $W_{j}^{g} \leftarrow W_{j}^{g}+W_{j}^{l}$. If the to-beremoved edge set $E$ is not empty, then those edges in other SPTs may be removed. For example, assume $\left(n_{j}, W_{j}^{g}[x]\right) \in$ $E$. If $n_{j} \in V\left(M^{i}\right)$ then edge $W_{j}^{g}[k]$ and all its downstream edges in its SPT will be removed.

When all nodes' local weight set is merged into their global weight set, all orphan nodes (those nodes removed during the voting process) will be attached to corresponding SPTs. In our approach, orphan nodes only attach to nodes who have minimum latency.

\section{EXPERIMENTAL EVALUATION}

In order to evaluate the performance of our proposed algorithm in a real networked virtual environment, we implemented a comprehensive simulation based on avatar mobility traces [6] collected from Second Life. The proposed algorithm was applied in two most popular regions: Freebies (slightly clustered) and Pharm (significantly clustered), as shown in Figures 3(a) and 3(b). In Freebies, avatars are slightly clustered in the land, while avatars are significantly clustered in Pharm land.

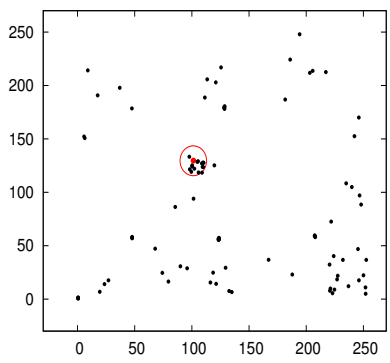

(a) Freebies

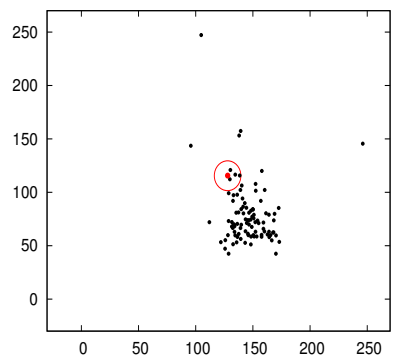

(b) Pharm
Figure 3: Snapshot of node positions in Second Life.

The end-to-end latency between any two nodes was randomly chosen from collected all-pair-ping data from PlanetLab. Realistic bandwidth limits for each node was randomly chosen from data gathered by dslreport.com. Every node will allocate $25 \%$ of its upload bandwidth to forward audio streams for others. The bandwidth unit that is required for every audio stream was set to $25 \mathrm{kbps}$. An AoI radius was assigned to all nodes and set to $10 \mathrm{~m}$. To make multicast trees adapt to node dynamics, newcomers randomly chose an existing node that had minimum latency as their parent. Once the ratio of receivers with minimum latency was less than a given threshold (we choose $25 \%$ in our simulation), the multicast tree was re-constructed.

The first group of the simulation results demonstrates that the proposed approach will achieve a high ratio of successful receivers versus the total number of candidate receivers. In CTA, the number of receivers that have minimum latency from their source is maximized at each time when a new multicast tree is constructed. Therefore, it will result in more nodes that have minimum latency, as shown in Figures 5(a) and 5(b). Furthermore, the orphan nodes of the newly constructed SPT have high probability to be attached to the SPT. As illustrated in Figures 4(a) and 4(b), the proposed approach outperforms the approach in [8], especially in Pharm land where avatars are very close to each other. 


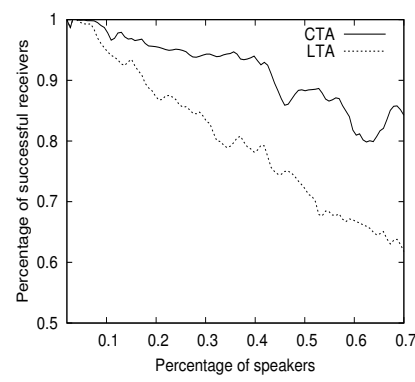

(a) Freebies

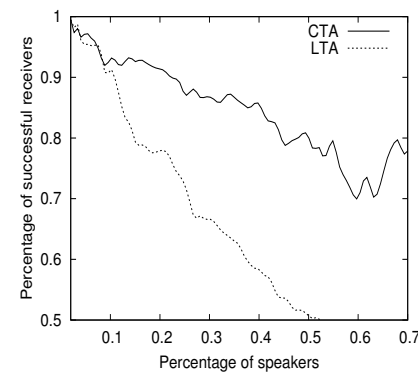

(b) Pharm
Figure 4: Ratio of successful receivers versus percentage of speakers.

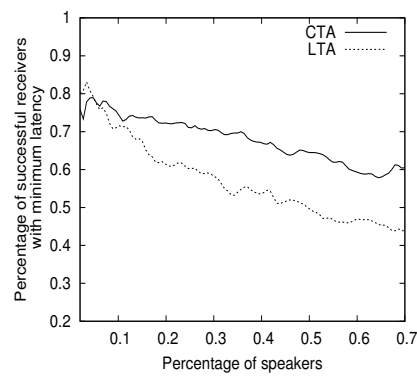

(a) Freebies

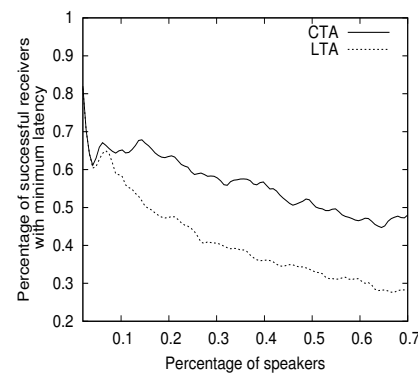

(b) Pharm
Figure 5: Ratio of successful receivers with minimum latency versus percentage of speakers.

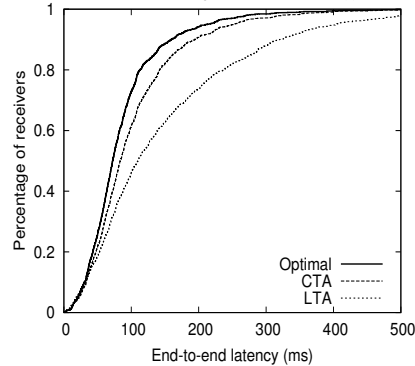

(a) Freebies

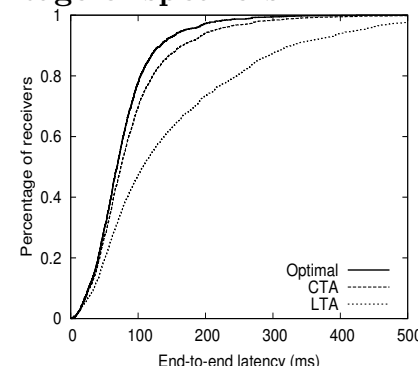

(b) Pharm
Figure 6: CDF of latency when there are $20 \%$ speakers in both lands.

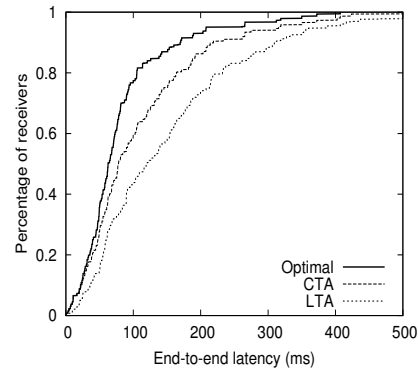

(a) Freebies

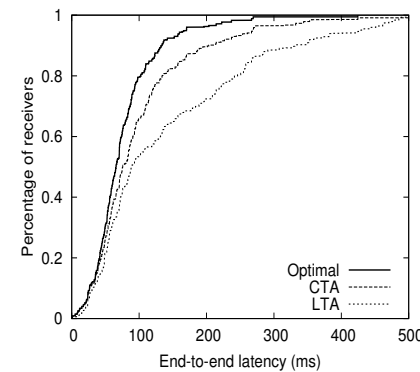

(b) Pharm
Figure 7: CDF of latency when there are 50\% speakers in both lands..

The second group of the simulation results shows the cumulative distribution function of receivers' latency in Freebies land and Pharm land when there are 20\% and $50 \%$ speakers in both lands. The optimal curve stands for the case when all receivers have minimum latency. We can see from the results that CTA has a lower overall latency compared with LTA.

\section{CONCLUSION}

In this paper, we introduced a heuristic algorithm, termed CTA, to adjust multiple multicast trees for spatialized audio streaming in NVEs. The main motivation of our work is the desire to quickly deploy multicast trees for speakers and maximize the number of successful receivers while at the same time keeping the average latency as low as possible. With the proposed algorithm we incrementally construct and adjust a multicast tree for each speaker. The proposed algorithm was employed in a simulation environment with real avatar mobility traces collected from Second Life. The simulation results show that CTA can achieve good performance both in terms of a large proportion of successful receivers that have minimum latency and also a relatively low overall latency. We are currently working on performing realistic measurements with a prototype implementation.

\section{ACKNOWLEDGEMENT}

This research has been funded in part by NSF grant IIS0534761 and A*STAR grant 0821010028 . We are grateful to Wei Tsang Ooi for providing us with avatar mobility traces. We also acknowledge the support of the NUS Interactive and Digital Media Institute (IDMI).

\section{REFERENCES}

[1] S. Banerjee, C. Kommareddy, K. Kar, B. Bhattacharjee, and S. Khuller. Construction of an Efficient Overlay Multicast Infrastructure for Real-Time Applications. Twenty-Second Annual Joint Conference of the IEEE Computer and Communications Societies, 2:1521-1531 vol.2, April 2003.

[2] A. Blum, P. Chalasani, D. Coppersmith, B. Pulleyblank, P. Raghavan, and M. Sudan. The Minimum Latency Problem. In STOC '94: Proceedings of the Twenty-Sixth Annual ACM Symposium on Theory of Computing, pages 163-171, New York, NY, USA, 1994.

[3] P. Boustead, F. Safaei, and M. Dowlatshahi. Dice: Internet Delivery of Immersive Voice Communication for Crowded Virtual Spaces. VR' 05: Virtual Reality., pages 35-41, March 2005.

[4] J. R. Douceur, J. R. Lorch, and T. Moscibroda. Maximizing Total Upload in Latency-Sensitive P2P Applications. In SPAA '0\%: Proceedings of The Nineteenth Annual ACM Symposium on Parallel Algorithms and Architectures., pages 270-279, New York, NY, USA, 2007.

[5] M. T. Helmick and F. S. Annexstein. Depth-Latency Tradeoffs in Multicast Tree Algorithms. AINA '07: Advanced Information Networking and Applications., pages 555-564, May 2007.

[6] H. Liang, I. Tay, M. F. Neo, W. T. Ooi, and M. Motani. Avatar Mobility in Networked Virtual Environments: Measurements, Analysis, and Implications. arXiv:0807.2328v1 2008.

[7] C. Nguyen, F. Safaei, and P. Boustead. Performance Evaluation of a Proxy System for Providing Immersive Audio Communication to Massively Multi-player Games. GlobeCom '04: Global Telecommunications Conference Workshops., pages 192-199, Dec. 2004.

[8] R. Zimmermann and K. Liang. Spatialized Audio Streaming for Networked Virtual Environments. In $M M$ '08: Proceeding of the 16th ACM International Conference on Multimedia, pages 299-308, New York, NY, USA, 2008.

[9] R. Zimmermann, B. Seo, L. S. Liu, R. S.Hampole, and B. Nash. Audiopeer: A Collaborative Distributed Audio Chat System. Distributed Multimedia Systems, San Jose, $C A, 2004$ 